\title{
Comparison of long-term survival between temozolomide-based chemoradiotherapy and radiotherapy alone for patients with low-grade gliomas after surgical resection
}

\author{
This article was published in the following Dove Press journal: \\ OncoTargets and Therapy \\ 17 August 2016 \\ Number of times this article has been viewed
}

\section{Xiu-juan Gai ${ }^{1,2}$ \\ Yu-mei Wei ${ }^{2}$ \\ Heng-min $\mathrm{TaO}^{1,2}$ \\ Dian-zheng $\mathrm{An}^{2}$ \\ Jia-teng Sun ${ }^{1,2}$ \\ Bao-sheng $\mathrm{Li}^{2}$}

'School of Medicine and Life Sciences, University of Jinan-Shandong Academy of Medical Sciences, Jinan, Shandong, People's Republic of China; ${ }^{2}$ Department of Radiation Oncology VI, Shandong Cancer Hospital Affiliated to Shandong University, Jinan, Shandong,

People's Republic of China
Correspondence: Bao-sheng Li

Department of Radiation Oncology VI, Shandong Cancer Hospital Affiliated to Shandong University, 440 Jiyan Road, Jinan, Shandong 250II7,

People's Republic of China

Tel +86 I86 I5I93697

Email baoshli1963@163.com
Purpose: This study was designed to compare the survival outcomes of temozolomide-based chemoradiotherapy $(\mathrm{TMZ}+\mathrm{RT})$ vs radiotherapy alone (RT-alone) for low-grade gliomas (LGGs) after surgical resection.

Patients and methods: In this retrospective analysis, we reviewed postoperative records of 69 patients with LGGs treated with TMZ + RT $(n=31)$ and RT-alone $(n=38)$ at the Shandong Cancer Hospital Affiliated to Shandong University between June 2011 and December 2013. Patients in the TMZ + RT group were administered 50-100 mg oral TMZ every day until the radiotherapy regimen was completed.

Results: The median follow-up since surgery was 33 months and showed no significant intergroup differences $(P=0.06)$. There were statistically significant intergroup differences in the progression-free survival rate $(P=0.037)$, with $83.9 \%$ for TMZ-RT group and $60.5 \%$ for RT-alone group. The overall 2-year overall survival (OS) rate was $89.86 \%$. Age distribution ( $\geq 45$ years and $<45$ years) and resection margin (complete resection or not) were significantly associated with $\mathrm{OS}(P=0.03$ and $P=0.004$, respectively).

Conclusion: Although no differences were found in the 2-year OS between the TMZ + RT and RT-alone groups, there was a trend toward increased 2-year progression-free survival in the TMZ + RT group. With better tolerability, concurrent TMZ chemoradiotherapy may be beneficial for postoperative patients with LGGs. Age distribution and surgical margin are likely potential indicators of disease prognosis. The possible differences in long-term survival between the two groups and the links between prognostic factors and long-term survival may be worthy of further investigation.

Keywords: low-grade gliomas, concurrent chemoradiotherapy, temozolomide (TMZ), radiotherapy alone, postoperative patients

\section{Introduction}

Low-grade gliomas (LGGs) include Grade II astrocytomas, oligodendrogliomas, and oligoastrocytomas. ${ }^{1}$ Surgery is usually the first choice of treatment. It is as still unclear whether the best option for postoperative patients with LGGs is radiotherapy (RT) alone or chemoradiotherapy. A Phase III trial investigated differences in patients with LGGs, who underwent incomplete resection and subsequent RT with or without chemotherapy (procarbazine or lomustine [CCNU]), but no statistically significant differences were observed owing to its premature termination. ${ }^{2}$ In another Phase III trial by the Radiation Therapy Oncology Group, RTOG 9802, ${ }^{3}$ a categorical benefit in 
overall survival (OS) was seen in the procarbazine, lomustine, and vincristine $(\mathrm{PCV})+\mathrm{RT}$ arm compared with the RTalone arm; however, the patients selected for this trial were not all of postoperative status and also included those who were $>40$ years old and had undergone a subtotal resection. ${ }^{3}$ This trial resulted in chemoradiotherapy being the standard treatment for high-risk LGG patients.

In recent times, temozolomide (TMZ) with its improved side effects has garnered much attention. TMZ is a secondgeneration alkylating agent with a low molecular mass. It has excellent oral bioavailability, attains peak plasma concentration in 1 hour, and easily penetrates the blood-brain barrier. In 2005, TMZ was considered as standard chemotherapy for glioblastoma treatment. Meanwhile, another trial, RTOG 0424, was initiated to identify the role of TMZ in conjunction with RT for postoperative LGGs. The recently published results showed that the 3-year OS rate of high-risk LGG patients who underwent TMZ chemotherapy plus RT was higher than that of the controls who only underwent RT. ${ }^{4}$ However, the trial was a single-arm study that used historical controls for comparison. Our retrospective study was designed to investigate the role of TMZ in combination with RT for postoperative LGGs.

\section{Patients and methods}

\section{Patient selection}

Between June 2011 and December 2013, 83 patients with pathologically confirmed Grade II gliomas (including astrocytomas, oligodendrogliomas, and mixed oligoastrocytomas) at the Shandong Cancer Hospital Affiliated to the Shandong University were retrospectively analyzed, but 14 patients were lost to follow-up. Thus, 69 patients were selected for the analysis. All patients were at least 18 years old at diagnosis. Besides surgery, no prior treatments were performed before radiation or chemoradiotherapy. Other diseases had to be evaluable. Patients with a history of other cancers who were in remission were excluded. Table 1 lists the main characteristics of the enrolled patients: 31 patients in the concurrent TMZ chemoradiotherapy group (TMZ + RT), and 38 patients in the radiation alone group (RT-alone). There were no statistically significant differences in patient features or tumor characteristics between the two groups (Table 1). The study was approved by the ethics committee of the Shandong Cancer Prevention and Treatment Research who deemed patient consent was not required due to the retrospective nature of the study.

\section{Radiotherapy}

RT was performed 2-4 weeks after surgery when the skin incision had almost healed. Patients were fixed with a thermal
Table I Patient characteristics for postoperative patients with LGG treated with concurrent TMZ + RT vs RT-alone ( $n=69)$

\begin{tabular}{|c|c|c|c|c|}
\hline Characteristic value & $\mathbf{T M Z}+\mathbf{R T}$ & RT-alone & Total & $P$-value \\
\hline Numbers of patient & 31 & 38 & 69 & \\
\hline Age (years) & & & & 0.33 \\
\hline Median & 41 & 43 & 43 & \\
\hline Range & $18-62$ & $24-63$ & $18-63$ & \\
\hline Age distribution & & & & 0.61 \\
\hline$\geq 45$ years & 12 & 17 & 29 & \\
\hline$<45$ years & 19 & 21 & 40 & \\
\hline Sex & & & & 0.65 \\
\hline Male & 18 & 20 & 38 & \\
\hline Female & 13 & 18 & 31 & \\
\hline Resection margin & & & & 0.30 \\
\hline Total & 22 & 31 & 53 & \\
\hline Subtotal & 9 & 7 & 16 & \\
\hline Histopathology & & & & 0.32 \\
\hline Astrocytomas & 17 & 27 & 44 & \\
\hline Oligodendrogliomas & 6 & 6 & 12 & \\
\hline Oligoastrocytomas & 8 & 5 & 13 & \\
\hline Time span (days) & & & & 0.86 \\
\hline Mean & 17 & 18 & & \\
\hline Range & $14-28$ & $16-25$ & & \\
\hline RT dose (Gy) & & & & 0.10 \\
\hline Median & 60 & 60 & 60 & \\
\hline Range & $50-66$ & $50-66$ & $50-66$ & \\
\hline
\end{tabular}

Notes: Resection margin: complete resection or incomplete, time span: from surgery to the start of radiotherapy.

Abbreviations: LGGs, low-grade gliomas; TMZ, temozolomide; RT, radiotherapy.

head mold and positioned under a computed tomography simulator. Radiation was delivered using a Varian linear accelerator (Varian Medical Systems, Palo Alto, CA, USA). The gross tumor volume was targeted on the contrastenhancing residual tumor and/or surgical residual cavity of postoperative T2-weighted magnetic resonance imaging. The planning target volume was more than $2 \mathrm{~cm}$ margin to the dose of $50 \mathrm{~Gy}$, and then the expanded margin was shrunk to $1 \mathrm{~cm}$ with a total dose ranging from 50 to $66 \mathrm{~Gy}$ in daily 2 Gy fractions by three-dimensional conformal radiation therapy..$^{5-7}$ Once symptoms of cerebral edema (such as headache, dizziness, or nausea) appeared during radiation, patients were given different doses of mannitol and dexamethasone by intravenous infusion based on the severity of symptoms.

\section{Chemotherapy}

Patients undergoing chemotherapy provided written consent for concurrent oral administration of 50-100 mg TMZ every day until the RT regimen was completed. Myelosuppressive and digestive-symptom side effects were effectively relieved. TMZ administration was stopped if the disease progressed or unacceptable toxicity was observed. Fortunately, only two patients in group TMZ + RT (2/31) showed 
slight digestive symptoms during therapy, and myelosuppression was not observed in anybody. No patient required cessation of concurrent TMZ chemotherapy owing to intolerable toxicity.

\section{Follow-up}

Follow-up was every 3 months in the first 2 years and then every 6 months thereafter. Each visit included physical examinations, magnetic resonance imaging of the brain, and other examinations according to symptoms (as necessary).

\section{Statistical analysis}

The following definitions were used for statistical analyses: progression-free survival (PFS) was defined as the time from the date of surgery to the date of disease progression or latest follow-up; OS was defined as the time from the date of surgery to the date of death or latest follow-up.

Differences in patient demographics, tumors, and treatment were calculated using Student's $t$-test for numerical data and $\chi^{2}$ test or Fisher's exact test for categorical data. Kaplan-Meier survival curves were plotted, and the survival distributions between the two groups were compared using the log-rank test. The Cox regression analysis was used to assess the association between OS or PFS and prognostic factors. Two-sided $P$-values of $<0.05$ were considered statistically significant. Statistical calculations were performed using SPSS version 17.0 statistical software (SPSS Inc., Chicago, IL, USA).

\section{Results}

Of all enrolled patients, 12 (38.71\%) and $17(43.58 \%)$ patients were $>45$ years in the TMZ + RT and RT-alone groups, respectively $(P=0.61)$. More patients in the $\mathrm{TMZ}+\mathrm{RT}$ group underwent a subtotal resection than those in the RT-alone group $(29.03 \%$ vs $18.42 \%)$, but this difference was not statistically significant $(P=0.30)$. The median follow-up after surgery was 33 months (range, 10-72 months) and was similar between the two groups (29 months for the TMZ + RT group and 35.5 months for RT-alone group; $P=0.06$ ). The overall 2-year PFS rate was $71.0 \%$ (83.9\% for TMZ-RT group and $60.5 \%$ for RT-alone group). The subgroups showed statistically significant differences ( $P=0.037$, Figure 1$)$. The overall 2-year OS rate was $89.86 \%$ (Figure 2). The median survival time has not yet been reached.

We conducted a Cox regression analysis to study the association between OS or PFS and the following prognostic factors: chemotherapy (with TMZ), age, age distribution ( $\geq 45$ years old and $<45$ years old), sex, resection margin (complete or incomplete), radiation dose, and histology.

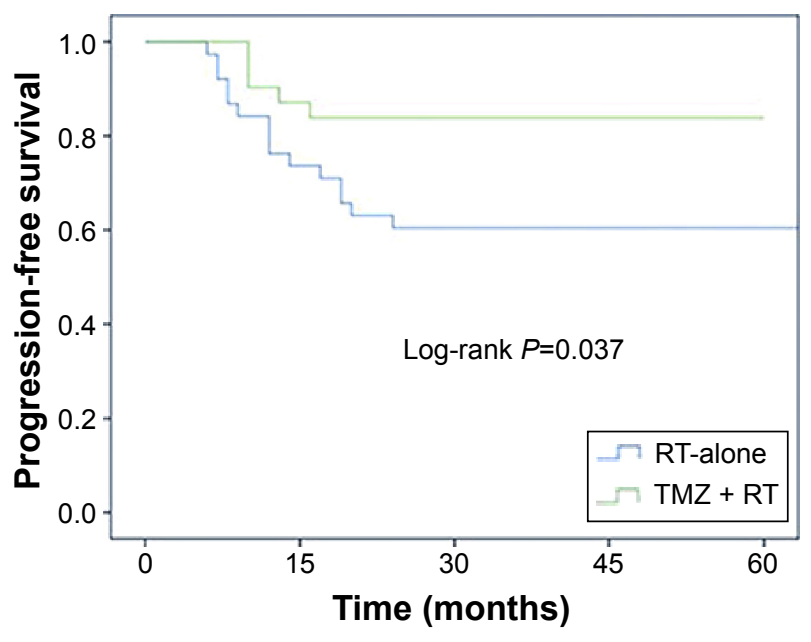

Figure I Comparison of progression-free survival for TMZ-RT and RT-alone groups.

Abbreviations: TMZ, temozolomide; RT, radiotherapy.

Age distribution and resection margin were significantly associated with OS $(P=0.03$, hazard ratio $[\mathrm{HR}]=0.015,95 \%$ confidence interval (CI): 0.00-0.65; $P=0.004, \mathrm{HR}=19.89$, 95\% CI: 2.65-149.29, respectively). The concurrent TMZ chemotherapy was significantly associated with PFS $(P=0.046, \mathrm{HR}=0.34,95 \%$ CI: $0.12-0.98$; Table 2$)$.

\section{Discussion}

The role of chemotherapy in patients with LGGs had not been clearly established until the results of the RTOG 9802 Phase III trial were published. Previously, recurrent or progressive LGGs treated with single agents such as nitrosourea or TMZ or with combined chemotherapy such as CCNU and PCV showed encouraging responses, ${ }^{8-14}$ but no convincing OS benefit. ${ }^{13,15}$ Then, the results of the RTOG 9802 trial $^{3}$ showed significant improvements in PFS among patients treated

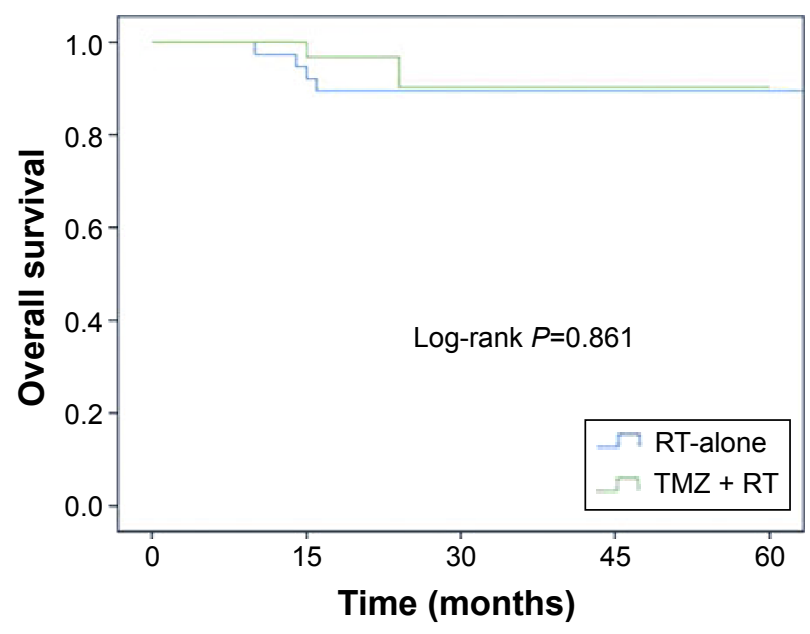

Figure 2 The 2-year OS rate for TMZ-RT and RT-alone groups. Abbreviations: TMZ, temozolomide; RT, radiotherapy; OS, overall survival. 
Table 2 Association between PFS or OS and the prognostic factors for postoperative patients with LGGs treated with TMZ + RT or RT-alone $(n=69)$

\begin{tabular}{lll}
\hline $\begin{array}{l}\text { Prognostic } \\
\text { factors }\end{array}$ & \multicolumn{1}{l}{$\boldsymbol{P}$-value HR (95\% CI) } \\
\cline { 2 - 3 } Sex & PFS & OS \\
Age & $0.58(0.76,0.29-1.98)$ & $0.2 \mathrm{I}(0.28,0.04-2.06)$ \\
Age distribution & $0.52(0.98,0.90-1.05)$ & $0.44(0.94,0.8 \mathrm{I}-\mathrm{I} .10)$ \\
Resection margin & $0.22(\mathrm{I} .95,0.08-2.62)$ & $0.03(0.015,0.00-0.65)$ \\
Radiation dose & $0.40(\mathrm{I} .06,0.93-\mathrm{I} .2 \mathrm{I})$ & $0.30(\mathrm{I} .2 \mathrm{I}, 0.84-\mathrm{I} .74)$ \\
Histology & $0.83(0.92,0.44-\mathrm{I} .93)$ & $0.67(0.72,0.16-3.23)$ \\
Chemotherapy & $0.046(0.34,0.12-0.98)$ & $0.375(0.45,2.65-149.3)$ \\
\hline
\end{tabular}

Notes: Age distribution: $\geq 45$ years old or not, resection margin: complete resection or incomplete, chemotherapy: with TMZ or not.

Abbreviations: LGGs, low-grade gliomas; TMZ, temozolomide; RT, radiotherapy; OS, overall survival; PFS, progression-free survival; $\mathrm{HR}$, hazard ratio; $\mathrm{Cl}$, confidence interval.

with PCV + RT. On account of its easy administration and a favorable quality-of-life profile, ${ }^{16} \mathrm{TMZ}$ has been studied more extensively in recent years. A few trials have demonstrated its efficacy by analyzing recurrent Grade II and III gliomas. ${ }^{8}{ }^{10}$ Some researchers report that chemotherapy could have been used to decrease the postoperative tumor volume for patients who underwent a subtotal resection, which was aimed at achieving a better prognosis. ${ }^{17-19}$ The RTOG 0424 trial ${ }^{4}$ focused on the survival benefit for postoperative patients treated with TMZ + RT compared with RT alone. However, patients in this randomized trial were required to have three or more risk factors for recurrence. Compared with the historical controls, patients showed a significantly improved 3 -year OS rate (54\% vs $73.1 \% ; P<0.001)$. The 3 -year PFS rate was $59.2 \%$. Despite not having such a long follow-up, an improved 2-year PFS rate of $83.9 \%(P=0.037)$ and 2-year OS rate of $90.3 \%(P=0.861)$ was recorded in our study. The reason for the insignificant 2-year OS rate may be attributed to the inclusion of patients with risk factors for recurrence in the RTOG 0424 trial, while our study excluded such patients. Furthermore, few patients in the TMZ + RT group selected TMZ as an adjuvant chemotherapy. However, all patients included in the RTOG 0424 trial were administered TMZ as the adjuvant chemotherapy. Moreover, as mentioned, TMZ was also found beneficial for recurrent gliomas. ${ }^{8,10}$ Once progression or recurrence was diagnosed, patients in both groups of our study would have gone on with continued therapies, with TMZ included. While these factors could collectively result in less obvious survival advantages, improvements in PFS and OS appear favorable.

On the other hand, prognosis factors identified by Pignatti et $\mathrm{al}^{20}$ were also included in the RTOG 0424 trial, and these were as follows: age $\geq 40$ years, astrocytoma histology, bihemispheric tumor, preoperative tumor diameter of $\geq 6 \mathrm{~cm}$, or a preoperative neurological function status of $>1$. Histology was the only factor significantly associated with OS $(P=0.027)$ and PFS $(P=0.034)$. Other reports also showed that the prognosis of oligodendrogliomas was better than astrocytomas of the same grade, with mixed gliomas featuring somewhere in between. ${ }^{20-22}$ However, histology did not show a significant association with either OS or PFS in our study. Meanwhile, we found that age distribution was also a related factor of OS $(P=0.03)$, but not of PFS $(P=0.34)$. The result should be viewed with the following considerations: 1) while patients in the RTOG 0424 trial underwent a subtotal resection, it was not considered a prognostic factor for OS; however, this was an important factor for OS $(P=0.004)$ in our study; 2) all patients in the RTOG 0424 trial had high-risk LGGs, including those $\geq 40$ years old; and 3 ) both our sample size and follow-up duration were comparatively shorter.

A growing number of studies have supported the role of surgical resection. ${ }^{23-25}$ After Jakola et $\mathrm{al}^{26}$ reported the comparison between surgery and biopsy, surgery became the first treatment choice for LGGs. No randomized trials examined the effect of the extent of surgical resection on PFS and OS, but its importance has been stressed by many recently published articles. A study with 170 patients with LGGs undergoing surgery showed a $95 \% 5$-year OS rate with almost total removal compared with $70 \%$ with the subtotal removal. ${ }^{27}$ Similarly, a study of 216 patients also found that the extent of resection was significantly related to OS: a 5-year OS rate of $76 \%$ associated with $<90 \%$ resection as compared with a 5 -year OS rate of $97 \%$ associated with $>90 \%$ resection. ${ }^{28}$ On the other hand, some studies show that although maximized surgery predicted improved OS in a univariate analysis, this association was lost when data were controlled for other prognostic factors. ${ }^{20}$ Our study found statistically significant differences in total and subtotal resection subgroups by using both univariate $(P=0.001)$ and multivariate analyses $(P=0.004)$.

In terms of radiation, no advantages for higher vs lower doses were seen, ${ }^{5,6}$ although a better survival for doses greater than 50 Gy has been reported. ${ }^{7}$ Most patients in our study were administered a dose $>50 \mathrm{~Gy}$, except for seven patients who received exactly $50 \mathrm{~Gy}$. Radiation dose was not related to either OS or PFS.

\section{Conclusion}

Concurrent TMZ chemoradiotherapy with better tolerability may be beneficial for postoperative patients with LGGs. In addition, age distribution and surgical margin could be 
used to indicate prognosis. Further studies are necessary to expand the reach of our observations and clarify the links between prognostic factors and long-term survival.

\section{Acknowledgments}

Contract grant sponsor: National Natural Science Foundation of China; contract grant number: 81530060. Contract grant sponsor: Natural Science Foundation of Shandong Province, People's Republic of China; contract grant number: ZR2015QZ09.

\section{Disclosure}

The authors report no conflicts of interest in this work.

\section{References}

1. Louis DN, Ohgaki H, Wiestler OD, et al. The 2007 WHO classification of tumours of the central nervous system. Acta Neuropathol. 2007;114:97-109.

2. Eyre HJ, Crowley JJ, Townsend JJ, et al. A randomized trial of radiotherapy versus radiotherapy plus CCNU for incompletely resected low-grade gliomas: a Southwest Oncology Group study. J Neurosurg. 1993;78:909-914.

3. Shaw E, Wang M, Coons SW, et al. Randomized trial of radiation therapy plus procarbazine, lomustine and vincristine chemotherapy for supratentorial adult low-grade glioma: Initial results of RTOG 9802. J Clin Oncol. 2012;30:3065-3070.

4. Fisher BJ, Hu C, Macdonald DR, et al. Phase 2 study of temozolomidebased chemoradiation therapy for high-risk low-grade gliomas: preliminary results of radiation therapy oncology group 0424. Int J Radiat Oncol Biol Phys. 2015;91:497-504.

5. Karim AB, Maat B, Hatlevoll R, et al. A randomized trial on doseresponse in radiation therapy of low-grade cerebral glioma: European Organization for Research and Treatment of Cancer (EORTC) Study 22844. Int J Radiat Oncol Biol Phys. 1996;36:549-556.

6. Shaw E, Arusell R, Scheithauer B, et al. Prospective randomized trial of low-versus high-dose radiation therapy in adults with supratentorial low-grade glioma: initial report of a North Central Cancer Treatment Group/Radiation Therapy Oncology Group/Eastern Cooperative Oncology Group study. J Clin Oncol. 2002;20:2267-2276.

7. Leighton C, Fisher B, Macdonald D, Stitt L, Bauman G, Cairncross J. The dose-volume interaction in adult supratentorial low-grade glioma: higher radiation dose is beneficial among patients with partial resection. J Neurooncol. 2007;82:165-170.

8. Quinn JA, Reardon DA, Friedman AH, et al. Phase II trial of temozolomide in patients with progressive or recurrent low-grade glioma. J Clin Oncol. 2003;21:646-651.

9. van den Bent MJ, Chinot O, Boogerd W, et al. Second line chemotherapy with temozolomide in recurrent oligodendroglioma after PCV (procarbazine, CCNU, vincristine) chemotherapy: EORTC Brain Tumor Group phase II study 26972. Ann Oncol. 2003;14:599-602.
10. Kesari S, Schiff D, Drappatz J, et al. Phase II study of protracted daily temozolomide for low-grade gliomas in adults. Clin Cancer Res. 2009; 15:330-337.

11. Kaloshi G, Benouaich-Amiel A, Diakite F, et al. Temozolomide for low-grade gliomas: predictive impact of $1 \mathrm{p} / 19 \mathrm{q}$ loss on response and outcome. Neurology. 2007;68:1831-1836.

12. Galanis E, Buckner JC, Burch PA, et al. Phase II trial of nitrogen mustard, vincristine and procarbazine (MOP) in patients with recurrent glioma: North Central Cancer Treatment Group results. J Clin Oncol. 1998;16:2953-2958.

13. Jemal A, Siegel R, Ward E, et al. Cancer statistics. CA Cancer J Clin. 2006;56:106-130.

14. Taal W, Dubbink HJ, Zonnenberg CB, et al. First-line temozolomide chemotherapy in progressive low-grade astrocytomas after radiotherapy: molecular characteristics in relation to response. Neuro Oncol. 2011;13:235-241.

15. Mason WP, Krol GS, DeAngelis LM. Low grade oligodendroglioma responds to chemotherapy. Neurology. 1996;46:203-207.

16. Liu R, Solheim K, Polley MY, et al. Quality of life in low-grade glioma patients receiving temozolomide. Neuro Oncol. 2009;11:59-68.

17. Kaloshi G, Benouaich-Amiel A, Diakite F, et al. Temozolomide for low-grade gliomas: predictive impact of $1 \mathrm{p} / 19 \mathrm{q}$ loss on response and outcome. Neurology. 2007;68:1831-1836.

18. Bello L, Gallucci M, Fava M, et al. Intraoperative subcortical language tract mapping guides surgical removal of gliomas involving speech areas. Neurosurgery. 2007;60:67-80; discussion 80-82.

19. Ricard D, Kaloshi G, Amiel-Benouaich A, et al. Dynamic history of low-grade gliomas before and after temozolomide treatment. Ann Neurol. 2007;61:484-490.

20. Pignatti F, van den Bent M, Curran D, et al. Prognostic factors for survival in adult patients with cerebral low-grade glioma. J Clin Oncol. 2002; 20:2076-2084

21. Deb P, Sharma MC, Mahapatra AK, Agarwal D, Sarkar C. Glioblastoma multiforme with long-term survival. Neurol India. 2005;53:329-332.

22. Jaeckle KA, Decker PA, Ballman KV, et al. Transformation of low grade glioma and correlation with outcome: an NCCTG database analysis. J Neurooncol. 2011;104:253-259.

23. Smith JS, Chang EF, Lamborn KR, et al. Role of extent of resection in the long-term outcome of low-grade hemispheric gliomas. J Clin Oncol. 2008;26:1338-1345.

24. Claus EB, Horlacher A, Hsu L, et al. Survival rates in patients with lowgrade glioma after intraoperative magnetic resonance image guidance. Cancer. 2005; 103:1227-1233.

25. Sanai N, Berger MS. Extent of resection influences outcomes for patients with gliomas. Rev Neurol (Paris). 2011;167:648-654.

26. Jakola AS, Myrmel KS, Kloster R, et al. Comparison of strategy favoring early surgical resection vs a strategy favoring watchful waiting in low-grade gliomas. JAMA. 2012;308:1881-1888.

27. McGirt MJ, Chaichana KL, Attenello FJ, et al. Extent of surgical resection is independently associated with survival in patients with hemispheric infiltrating low-grade gliomas. Neurosurgery. 2008;63:700-707.

28. Smith JS, Chang EF, Lamborn KR, et al. Role of extent of resection in the long-term outcome of low-grade hemispheric gliomas. $J$ Clin Oncol. 2008;26:1338-1345.
OncoTargets and Therapy

\section{Publish your work in this journal}

OncoTargets and Therapy is an international, peer-reviewed, open access journal focusing on the pathological basis of all cancers, potential targets for therapy and treatment protocols employed to improve the management of cancer patients. The journal also focuses on the impact of management programs and new therapeutic agents and protocols on

\section{Dovepress}

patient perspectives such as quality of life, adherence and satisfaction. The manuscript management system is completely online and includes a very quick and fair peer-review system, which is all easy to use. Visit http://www.dovepress.com/testimonials.php to read real quotes from published authors. 